\title{
INTEGRAL INEQUALITIES OF HARDY AND POINCARÉ TYPE
}

\author{
HAROLD P. BOAS AND EMIL J. STRAUBE
}

(Communicated by John B. Conway)

\begin{abstract}
The Poincaré inequality $\|u\|_{p} \leq C\|\nabla u\|_{p}$ in a bounded domain holds, for instance, for compactly supported functions, for functions with mean value zero and for harmonic functions vanishing at a point. We show that it can be improved to $\|u\|_{p} \leq C\left\|\delta^{\beta} \nabla u\right\|_{p}$, where $\delta$ is the distance to the boundary, and the positive exponent $\beta$ depends on the smoothness of the boundary.
\end{abstract}

1. Introduction. In this note we improve standard versions of Poincarés inequality by applying Hardy's inequality for bounded domains $\Omega$ in $\mathbf{R}^{n}$. Our work was stimulated by a recent paper of Ziemer in which he showed $[\mathbf{Z}, \S 3]$ that if the boundary of $\Omega$ is locally the graph of a continuous function, then for every linear second-order elliptic equation there is a constant $C$ such that

$$
\|u\|_{p} \leq C\|\nabla u\|_{p}
$$

for every solution $u$ normalized by $u\left(x_{0}\right)=0$. Here $\|u\|_{p}$ is the $L^{p}(\Omega)$ norm of $u$ $(1 \leq p<\infty)$ and $\nabla$ denotes the gradient. We show that if the boundary of $\Omega$ is locally the graph of a Hölder continuous function of exponent $\alpha$ then the right-hand side of (1.1) can be replaced by $C\left\|\delta^{\alpha} \nabla u\right\|_{p}$, where $\delta$ denotes the distance to the boundary. A similar improvement of Poincaré's inequality holds for many other function classes; see $\S 2$.

We denote the space of functions $u$ with norm $\|u\|_{p}+\left\|\delta^{\alpha} \nabla u\right\|_{p}<\infty$ by $W^{1, p}(\Omega, \alpha)$, or just $W^{1, p}(\Omega)$ when $\alpha=0$, while $W_{\text {loc }}^{1, p}(\Omega)$ denotes the space of functions that lie in $W^{1, p}(\omega)$ for every $\omega \Subset \Omega$. Our abstract version of Poincaré's inequality is the following

THEOREM. Let $\Omega$ be a bounded domain in $\mathbf{R}^{n}$ whose boundary is locally the graph of a Hölder continuous function of exponent $\alpha$, where $0 \leq \alpha \leq 1$, and suppose $1 \leq p<\infty$. Let $H$ be a cone in $W_{\text {loc }}^{1, p}(\Omega)$ such that the closure of $H \cap W^{1, p}(\Omega, \alpha)$ in $W^{1, p}(\Omega, \alpha)$ contains no nonzero constant function. Then there is a constant $C$ such that

$$
\|u\|_{p} \leq C\left\|\delta^{\alpha} \nabla u\right\|_{p}
$$

for every function $u$ in $H$, where $\delta$ denotes the distance to the boundary of $\Omega$.

It is part of the Theorem that finiteness of the right-hand side implies finiteness of the left-hand side. When $\alpha=0$ we understand the boundary of $\Omega$ to be locally

Received by the editors January 20, 1987.

1980 Mathematics Subject Classification (1985 Revision). Primary 46E35; Secondary 26D10, $35 \mathrm{H} 05$.

Key words and phrases. Poincaré's inequality, Hardy's inequality, hypoelliptic partial differential equations.

The first author was partially supported by NSF grant DMS-8501758. 
the graph of simply a continuous function, and we recapture (1.1) with no gain. When $\alpha=1$ the boundary of $\Omega$ is locally the graph of a Lipschitz function-this is equivalent $[G$, Theorem 1.2.2.2] to the uniform cone condition for $\Omega$, and holds, for instance, for every convex domain - and we attain the maximal gain of a full power of $\delta$ in (1.2).

We give the proof of the Theorem in $\S 3$. In $\S 2$ we obtain improvements of various standard formulations of Poincaré's inequality by specifying the cone $H$. In $\S 4$ we show that the Theorem is sharp and also indicate some extensions.

\section{Examples.}

EXAMPLE 2.1. Suppose $\int_{\Omega} \phi d V \neq 0$ for a function $\phi$ in $L^{q}(\Omega)$, where $p^{-1}+$ $q^{-1}=1$. The set $H:=\left\{u \in L^{p}(\Omega) \cap W_{\text {loc }}^{1, p}(\Omega): \int_{\Omega} u \phi d V=0\right\}$ satisfies the assumptions of the Theorem. In particular, the Theorem applies to the class of functions with mean value zero (take $\phi$ to be identically one).

EXAMPLE 2.2. The set $H:=\left\{u \in W_{\text {loc }}^{1, p}(\Omega): u\right.$ vanishes on a set of measure at least $\gamma$ \} satisfies the assumptions of the Theorem, where $\gamma$ is a fixed positive number.

EXAMPLE 2.3. Suppose $p>n$. In this case, $W_{\text {loc }}^{1, p}(\Omega)$ embeds in the space of continuous functions by Sobolev's lemma [Ad, Theorem 5.4], so if $x_{0}$ is a fixed point in $\Omega$ the Theorem applies with $H:=\left\{u \in W_{\text {loc }}^{1, p}(\Omega): u\left(x_{0}\right)=0\right\}$.

EXAMPLE 2.4. Let $P$ be a linear partial differential operator on $\Omega$ with smooth coefficients. Suppose that $P$ is hypoelliptic; that is, every solution $u$ of the equation $P u=f$ is smooth on every open set where $f$ is. The Theorem applies with $H:=\left\{u \in W_{\mathrm{loc}}^{1, p}(\Omega): P u=0\right.$ and $\left.u\left(x_{0}\right)=0\right\}$. A nonzero constant cannot lie in the closure of $H \cap W^{1, p}(\Omega, \alpha)$ because, by the hypoellipticity of $P$ and the closed graph theorem, convergence in this space implies convergence in $C^{\infty}(\Omega)$.

The class of hypoelliptic operators contains, besides the elliptic operators, parabolic operators such as the heat operator, and also certain operators that arise in the theory of the $\bar{\partial}$-Neumann problem in several complex variables. See Chapter III and $\S 1$ of Chapter XV of [ $\mathbf{T}]$ for a discussion and further references.

EXAMPLE 2.5. Consider a linear second-order equation

$$
\sum_{i, j=1}^{n} \frac{\partial}{\partial x_{i}}\left(a_{i j} \frac{\partial u}{\partial x_{j}}+b_{i} u\right)+\sum_{i=1}^{n} c_{i} \frac{\partial u}{\partial x_{i}}+d u=0
$$

in divergence form, where the coefficients $a_{i j}, b_{i}, c_{i}$, and $d$ are only assumed to be locally bounded in $\Omega$, and suppose (2.1) is locally strictly elliptic in the sense that for every compact set $K \subset \Omega$ there is a positive $\lambda$ such that

$$
\sum_{i, j=1}^{n} a_{i j}(x) \xi_{i} \xi_{j} \geq \lambda|\xi|^{2} \quad \text { for all } x \in K \text { and } \xi \in \mathbf{R}^{n}
$$

The Theorem applies with $H:=\left\{u \in W_{\mathrm{loc}}^{1,2}(\Omega): u\right.$ is a weak solution of $(2.1)$ and $\left.u\left(x_{0}\right)=0\right\}$. Indeed by Theorem 8.24 of $[\mathbf{G T}]$ there is some $\gamma>0$ for which

$$
\|u\|_{C^{\gamma}(\omega)} \leq C\|u\|_{L^{2}(\Omega)},
$$

where $x_{0} \in \omega \Subset \Omega$. Hence $H \cap W^{1,2}(\Omega, \alpha)$ contains no nonzero constant in its closure. The special case $\alpha=0$ is essentially the result of $\S 3$ of $[\mathbf{Z}]$. 
In the previous two examples we considered solutions of linear partial differential equations. Since the set $H$ is not required to be a subspace, but only a cone, certain homogeneous nonlinear partial differential equations are also within the scope of the Theorem. However, for general nonlinear equations the constant $C$ must be allowed to depend on the norm of $u$ : see $\S 2$ of $[\mathbf{Z}]$ where the case of second-order nonlinear elliptic equations is considered.

EXAMPLE 2.6. Suppose $H:=C_{0}^{\infty}(\Omega)$ is the space of smooth compactly supported functions in $\Omega$. The estimate $\left\|\delta^{-\alpha} u\right\|_{p} \leq\|\nabla u\|_{p}$ for $u$ in $C_{0}^{\infty}(\Omega)$ follows from [Kf, Theorem 8.4] and the classical Poincaré inequality, while some related inequalities with the weight entirely on the left-hand side are discussed in the recent papers [An and Le]. Our Theorem implies that part, but not all, of the weight can be moved to the right-hand side.

PROPOSITION. Let $\Omega$ be a bounded domain in $\mathbf{R}^{n}$ whose boundary is locally the graph of a Hölder continuous function of exponent $\alpha$, where $0<\alpha \leq 1$, and suppose $1<p<\infty$. Then for all $u$ in $C_{0}^{\infty}(\Omega)$,

$$
\begin{gathered}
\left\|\delta^{-1 / p} u\right\|_{p} \leq C\left\|\delta^{\alpha-1 / p} \nabla u\right\|_{p} \quad \text { if } 1 / p \leq \alpha<1 ; \\
\left\|\delta^{-\varepsilon-1 / p} u\right\|_{p} \leq C\left\|\delta^{1-\varepsilon-1 / p} \nabla u\right\|_{p} \quad \text { if } \alpha=1 \text { and } 0<\varepsilon \leq 1-1 / p .
\end{gathered}
$$

P ROOF. By applying the classical one-variable Hardy inequality [HLP, Theorem 330] along a direction transverse to the boundary, one obtains [Kf, Theorem 8.4]

$$
\left\|\delta^{-\beta} u\right\|_{p} \leq C\left(\left\|\delta^{\alpha-\beta} \nabla u\right\|_{p}+\left\|\delta^{\alpha-\beta} u\right\|_{p}\right),
$$

where $\beta=1 / p$ if $1 / p \leq \alpha<1$ and $\beta=\varepsilon+1 / p$ (for any $\varepsilon>0$ ) if $\alpha=1$. Hence the closure of $C_{0}^{\infty}(\Omega)$ in $W^{1, p}(\Omega, \alpha-\beta)$ contains no nonzero constant $u$, since the lefthand side of (2.4) is infinite for such a $u$. The Theorem implies $\|u\|_{p} \leq C\left\|\delta^{\alpha-\beta} \nabla u\right\|_{p}$ for $u$ in $C_{0}^{\infty}(\Omega)$, and combining this with (2.4) gives the result of the Proposition.

The weaker inequality $\|u\|_{p} \leq C\left\|\delta^{1-\varepsilon-1 / p} \nabla u\right\|_{p}$ appears in [Kd, Theorem 12.8] for $\Omega$ with twice differentiable boundary. It is interesting that this estimate cannot be improved; that is, removing the negative power of $\delta$ from the left-hand side does not make it possible to increase the power of $\delta$ on the right-hand side. This is immediate from the following result, which extends Proposition 9.10 of $[\mathbf{K f}]$ to the case $\beta=1-p^{-1}$.

LEMMA. Let $\Omega$ be a bounded domain in $\mathbf{R}^{n}$ whose boundary is locally the graph of a Lipschitz continuous function. Then $C_{0}^{\infty}(\Omega)$ is dense in $W^{1, p}(\Omega, \beta)$ if $\beta \geq$ $1-p^{-1}>0$.

PROOF. It suffices to find compactly supported functions approximating $u \in$ $C^{\infty}(\bar{\Omega})$, since this space is dense [Kf, proof of Theorem 7.2] in $W^{1, p}(\Omega, \beta)$, and so it is enough to consider the case $\beta=1-p^{-1}$. Let $\Delta(x)$ be a regularized distance function [St, p. 171] for $\Omega$. Let $\phi_{n}(t)$ be a smooth function on $\mathbf{R}$ that vanishes outside the interval $\left(4^{-n}, 2^{-n}\right)$ and closely approximates the function $(n t \log 2)^{-1}$ in $L^{p}\left(4^{-n}, 2^{-n}\right)$, and set $\psi_{n}(x)=\int_{0}^{\Delta(x)} \phi_{n}(t) d t$. Then $\psi_{n} \in C_{0}^{\infty}(\Omega)$ and $\psi_{n} u \rightarrow u$ in $W^{1, p}\left(\Omega, 1-p^{-1}\right)$ because $\psi_{n} \rightarrow 1$ in $L^{p}(\Omega)$ and $\int_{\Omega} \delta^{p-1}\left|\nabla \psi_{n}\right|^{p}=O\left(n^{1-p}\right) \rightarrow 0$; the latter follows from Fubini's theorem by first integrating locally in the direction transverse to the boundary. 
3. Proof of the Theorem. The proof results from combining Hardy's inequality with a compactness argument that goes back at least to Morrey $[\mathbf{M}, \mathbf{p}$. 83]. The version of Hardy's inequality that we need (using different weights from those above) is a special case of Theorem 8.2 of [Kf]: there exists a smoothly bounded relatively compact subdomain $\omega$ of $\Omega$ such that

$$
\|u\|_{p} \leq C\left(\left\|\delta^{\alpha} \nabla u\right\|_{p}+\|u\|_{L^{p}(\omega)}\right)
$$

for every locally integrable function $u$. The statement in [Kf] does not include the case $\alpha=0$ nor the case $p=1$, and the error term in (3.1) is given there as $\left\|\delta^{\alpha} u\right\|_{p}$, but the stronger statement given here is implicit in the proof.

In view of (3.1), to prove the Theorem we need only show that $\|u\|_{L^{p}(\omega)} \leq$ $C\left\|\delta^{\alpha} \nabla u\right\|_{p}$ for every function $u$ in $H$. If this estimate did not hold, there would be a sequence of functions $u_{j}$ in $H$ such that

$$
\left\|u_{j}\right\|_{L^{p}(\omega)}=1
$$

and

$$
\left\|\delta^{\alpha} \nabla u_{j}\right\|_{p}<1 / j
$$

In particular, the $u_{j}$ form a bounded sequence in $W^{1, p}(\omega)$, which embeds compactly in $L^{p}(\omega)$ by the Rellich-Kondrashov theorem [Ad, Theorem 6.2]. By passing to a subsequence we may assume that the $u_{j}$ converge in $L^{p}(\omega)$ to some limit $u$, and in view of (3.1) and (3.3) the convergence even takes place in $W^{1, p}(\Omega, \alpha)$. But $\|u\|_{L^{p}(\omega)}=1$ by (3.2), and the gradient of $u$ vanishes identically by (3.3). Hence $u$ is a nonzero constant, which contradicts the hypothesis on $H$ and proves the Theorem.

4. Further results. (1) The Theorem is sharp in the following three senses. Without the hypothesis that the boundary be locally a graph, estimate (1.1) may fail for function classes other than $C_{0}^{\infty}(\Omega)$ : see p. 521 of $[\mathbf{C H}],[\mathbf{H}]$, and Theorem 10 of [AS]. The exponent $\alpha$ in (1.2) cannot be increased: if $\Omega$ is the planar domain $\left\{(x, y): 0<x<1,|y|<x^{1 / \alpha}\right\}$, where $0<\alpha \leq 1$, and $\beta>\alpha$, then $1+\alpha^{-1} \leq$ $\gamma p<1+\alpha^{-1}+(\beta-\alpha) p / \alpha$ implies $\left\|\delta^{\beta} \nabla z^{-\gamma}\right\|_{p}<\infty$ but $\left\|z^{-\gamma}\right\|_{p}=\infty$. When $u$ is harmonic, an inequality $\|\delta \nabla u\|_{p} \leq C\|u\|_{p}$ in the reverse directions holds because of the subaveraging property of $|u|^{p}$ : see for instance [D, Lemma 1$]$.

(2) Analogous statements, with a loss of $\varepsilon$ in the power of $\delta$, can be proved for $p=\infty$ by replacing the Rellich-Kondrashov theorem with the Arzelà-Ascoli theorem and Hardy's inequality with the convergence of $\int_{\Omega} \delta^{-\beta}$ for $\beta<\alpha$. For instance, one obtains that if $u$ has mean value zero in a bounded convex domain $\Omega$, then $\|u\|_{\infty} \leq C\left\|\delta^{\beta} \nabla u\right\|_{\infty}$ for every $\beta<1$.

(3) By the same method one can establish weighted Poincare inequalities of the form $\left\|\delta^{\gamma} u\right\|_{p} \leq C\left\|\delta^{\beta} \nabla u\right\|_{p}$ with $0<\gamma<\beta$. Even certain weights more general than powers are admissible (cf. [Kf, $\S 12])$.

(4) If the operator $P$ in Example 2.4 satisfies the strong maximum principle and if $\Omega$ has (say) once continuously differentiable boundary (so that $\alpha=1$ ), then (1.2) can be refined to $\|u\|_{p} \leq C\|\delta X u\|_{p}$, where $X$ is any smooth vector field that is everywhere transverse to the boundary. It is easy to see (by integrating along integral curves of $X$ ) that $X u$ can replace $\nabla u$ in the generalized Hardy inequality (3.1). Hence if there were a sequence of functions $u_{j}$ in $H$ with $\left\|u_{j}\right\|_{L^{p}(\omega)}=1$ and 
$\left\|\delta X u_{j}\right\|_{p}<1 / j$ the $u_{j}$ would be a bounded set in $L^{p}(\Omega)$. By the hypoellipticity a subsequence converges in $C^{\infty}(\Omega)$ to a solution of $P u=0$ with $X u=0$. But if $u$ is constant along the integral curves of $X$ then $u$ takes its maximum in the interior and so is constant. We obtain a contradiction as in $\S 3$ since in this case a nonzero constant cannot lie in even the $L^{p}(\Omega)$ closure of $H$. This phenomenon has recently found applications in several complex variables.

\section{REFERENCES}

[Ad] R. A. Adams, Sobolev spaces, Academic Press, New York, 1975.

[An] A. Ancona, On strong barriers and an inequality of Hardy for domains in $\mathbf{R}^{n}$, J. London Math. Soc. 34 (1986), 274-290.

[AS] S. Axler and A. L. Shields, Univalent multipliers of the Dirichlet space, Michigan Math. J. 32 (1985), 65-80.

[CH] R. Courant and D. Hilbert, Methoden der mathematischen Physik, vol. 2, Springer-Verlag, Berlin, 1937.

[D] J. Detraz, Classes de Bergman de fonctions harmoniques, Bull. Soc. Math. France 109 (1981), 259-268.

[GT] D. Gilbarg and N. S. Trudinger, Elliptic partial differential equations of second order, 2nd ed., Springer-Verlag, Berlin and New York, 1983.

[G] P. Grisvard, Elliptic problems in nonsmooth domains, Pitman, Boston, Mass., 1985.

[HLP] G. H. Hardy, J. E. Littlewood, and G. Pólya, Inequalities, Cambridge Univ. Press, 1934.

[H] J. A. Hummel, Counterexamples to the Poincaré inequality, Proc. Amer. Math. Soc. 8 (1957), 207-210.

[Kd] L. D. Kudryavtsev, Direct and inverse imbedding theorems, Transl. Math. Monographs, Amer. Math. Soc. Providence, R.I., 1974.

[Kf] A. Kufner, Weighted Sobolev spaces, Wiley, New York, 1985.

[Le] J. Lewis, Uniformly fat sets, preprint.

[M] C. B. Morrey, Multiple integrals in the calculus of variations, Springer, New York, 1966.

[St] E. M. Stein, Singular integrals and differentiability properties of functions, Princeton Univ. Press, Princeton, N.J., 1970.

[T] M. E. Taylor, Pseudodifferential operators, Princeton Univ. Press., Princeton, N.J., 1981.

[Z] W. P. Ziemer, A Poincaré-type inequality for solutions of elliptic differential equations, Proc. Amer. Math. Soc. 97 (1986), 286-290.

Department of Mathematics, Texas A\&M University, College Station, TEXAS 77843 (Current address of both authors)

Department of Mathematics and Statistics, University of PitTsburgh, Pittsburgh, PenNSylVANia 15260 